\title{
Dores osteomusculares e o estresse percebido por docentes durante a pandemia da COVID-19
}

\author{
Musculoskeletal pain and perceived stress by teachers during the COVID-19 pandemic \\ Dolor musculoesquelético y estrés percibido por docentes durante la pandemia COVID-19
}

\section{Resumo}

Objetivo: Analisar a presença de dores osteomusculares e de estresse percebido em docentes universitários em tempos de pandemia da COVID-19. Métodos: Estudo transversal de análise quantitativa realizado com o corpo docente de uma instituição de ensino superior entre os meses de outubro de dezembro de 2020. A coleta de dados foi realizada por meio de um questionário sociodemográfico e profissional, do Questionário Nórdico de Sintomas Osteomusculares (QNSO) e da Escala de Percepção de Estresse-10 (PSS-10). Resultados: A maioria dos 51 participantes eram mulheres (64,7\%); com idade entre 31 e 49 anos $(59,6 \%)$; casados $(66,7 \%)$ e com filhos $(58,8 \%)$. Possuíam especialização e mestrado $(41,2 \%)$; dois empregos $(62,7 \%)$; carga horária total de mais 40 horas semanais $(41,2 \%)$; com renda mensal entre quatro e seis salários mínimos (35,3\%); e com tempo de trabalho na instituição de até cinco anos (58,8\%). A principal queixa osteomuscular dos docentes foi na região das costas, inclusive gatilho para a procura por atendimentos de saúde. Apenas os problemas relacionados aos cotovelos não os impediram de realizar suas atividades normais no último ano. Conclusão: O período de atividade remota aponta uma percepção de estresse acima da média em $45,1 \%$ dos participantes, estando diretamente relacionada à existência de distúrbios osteomusculares no pescoço e ombros nos últimos 12 meses.

Palavras-chave: COVID-19; Docentes; Dor osteomuscular; Estresse psicológico.

\begin{abstract}
Objective: To analyze the presence of musculoskeletal pain and perceived stress in university professors in times of COVID-19 pandemic. Methods: Cross-sectional study of quantitative analysis carried out with the faculty of a higher education institution between the months of October, December 2020. Data collection was performed using a sociodemographic and professional questionnaire, from the Nordic Musculoskeletal Questionnaire (QNSO) and the Stress Perception Scale-10 (PSS-10). Results: Most of the 51 participants were women (64.7\%); aged between 31 and 49 years $(59.6 \%)$; married $(66.7 \%)$ and with children $(58.8 \%)$. They had a specialization and a master's degree $(41.2 \%)$; two jobs (62.7\%); total workload of more than 40 hours per week $(41.2 \%)$; with monthly income between four and six minimum wages $(35.3 \%)$; and with working time in the institution of up to five years $(58.8 \%)$. The teachers' main musculoskeletal complaint was in the back region, including the trigger for seeking health care. Only the problems
\end{abstract}


related to the elbows did not prevent them from carrying out their normal activities in the last year. Conclusion: The period of remote activity indicates a perception of stress above the average in $45.1 \%$ of the participants, being directly related to the existence of musculoskeletal disorders in the neck and shoulders in the last 12 months.

Keywords: COVID-19; Faculty; Musculoskeletal pain; Stress psychological.

\section{Resumen}

Objetivo: Analizar la presencia de dolor musculoesquelético y estrés percibido en profesores universitarios en tiempos de pandemia de COVID-19. Métodos: Estudio transversal de análisis cuantitativo realizado con el profesorado de una institución de educación superior entre los meses de octubre, diciembre de 2020. La recolección de datos se realizó mediante un cuestionario sociodemográfico y profesional, del Cuestionario Nórdico Musculoesquelético (QNSO) y el Stress Escala de percepción-10 (PSS-10). Resultados: La mayoría de los 51 participantes fueron mujeres (64,7\%); edad entre 31 y 49 años $(59,6 \%)$; casados $(66,7 \%)$ y con hijos $(58,8 \%)$. Tenían una especialización y una maestría $(41,2 \%)$; dos empleos (62,7\%); carga de trabajo total de más de 40 horas semanales $(41,2 \%)$; con ingresos mensuales entre cuatro y seis salarios mínimos (35,3\%); y con jornada laboral en la institución de hasta cinco años $(58,8 \%)$. La principal queja musculoesquelética de los docentes se encontraba en la región posterior, incluido el detonante para la búsqueda de atención médica. Solo los problemas relacionados con los codos no les impidieron realizar sus actividades habituales en el último año. Conclusión: El período de actividad remota apunta a una percepción de estrés superior a la media en el $45,1 \%$ de los participantes, estando directamente relacionado con la existencia de trastornos musculoesqueléticos en cuello y hombros en los últimos 12 meses.

Palabras clave: COVID-19; Docentes; Dolor musculoesquelético; Estrés psicológico.

\section{Introdução}

O ano de 2020 será marcado na história pela pandemia do Coronavírus (SARS-CoV-2), causador da doença popularmente conhecida como COVID-19, a qual impôs mudanças drásticas no comportamento da humanidade, de forma geral. Em conformidade com dados fornecidos pela Organização Mundial da Saúde (OMS), em 11 de março de 2021, um ano após a caracterização pela OMS da COVID-19 como pandemia, os casos confirmados de pessoas contaminadas ultrapassavam 117 milhões no planeta. Na mesma data, o Brasil possuía 11.122.429 casos confirmados e 269.309 mortes (WHO, 2021).

Desde o início do atual surto, percebe-se a grande preocupação de toda comunidade internacional na busca de métodos para o controle e estagnação do avanço da doença nas diversas regiões do mundo, em virtude do seu alto poder de contaminação, além de diversos impactos causados aos diversos setores da saúde, economia, educação, política, desenvolvimento social, entre outros (Freitas, Napimoga \& Donalisio, 2020).

Com o intuito de amenizar a transmissibilidade do vírus e alcançar um melhor resultado no tratamento das pessoas contaminadas, a OMS, o Ministério da Saúde brasileiro e outras organizações nacionais e internacionais sugeriram a aplicação de planos de contingência de influenza e suas ferramentas, em virtude da similaridade clínica e epidemiológica entre o vírus da COVID-19 e de demais vírus respiratórios (Freitas, Napimoga \& Donalisio, 2020).

Nesse cenário, busca-se a cooperação de todos os países para interromper a disseminação do vírus através da estratégia de isolamento social, ou seja, evita-se o contato entre pessoas sintomáticas ou assintomáticas, a fim de interromper o ciclo de transmissão e, com isso, evitar a propagação do vírus e a transmissão local (Brasil, 2020).

Contudo, o distanciamento social impactou diretamente na vida das pessoas. Na área da educação não seria diferente, uma vez que o ensino é predominantemente presencial nas instituições públicas e privadas do Brasil. Diante disso, no que se refere ao ensino superior integrante do sistema federal de ensino, o Ministério da Educação (MEC) autorizou a substituição das aulas presenciais por aulas em meios digitais enquanto durar a situação de pandemia do novo coronavírus (Portaria nº 544, 2020).

A adaptação a esse novo cenário apresenta grandes dificuldades e limitações, levando a um desgaste físico e emocional nos docentes e comprometimento da qualidade do ensino fornecido pelas instituições. Isto porque a docência já traz consigo obstáculos que refletem negativamente na saúde mental e na execução do serviço com qualidade e satisfação de tais profissionais, em virtude dos grandes problemas enfrentados pela classe de trabalhadores na educação dentro do ensino presencial ou, nos dias 
atuais do ensino remoto, opção apresentada como alternativa ao isolamento social enfrentado pela pandemia da COVID-19 (Monteiro \& Souza, 2020).

Nas últimas décadas, percebe-se um elevado aumento no adoecimento de docentes e profissionais da educação, especificamente no que se refere a sofrimentos mentais, ligados diretamente a precariedade e as novas condições de trabalho, tais sofrimentos compreendem "um conjunto de manifestações do corpo e da psique como estresse, ansiedade, depressão e fadiga", contemporaneamente definidos como "mal-estar docente" (Tostes, et al., 2018, p. 90).

Dessa forma, questiona-se se a pandemia da COVID-19 gerou dores osteomusculares e estresse aos docentes universitários, diante da drástica mudança de rotina vivenciada.

O presente estudo se justifica pelo fato de a pandemia da COVID-19 ser um assunto atual e de extrema relevância na atualidade em nossa sociedade, sendo que irá verificar os desdobramentos do isolamento social em docentes do Centro Universitário do Cerrado (UNICERP), instituição de ensino privado, especificamente no que se refere a dores osteomusculares e estresse em virtude das diversas adaptações necessárias para a conclusão do ano letivo.

Nesse contexto, o objetivo desta pesquisa é analisar a presença de dores osteomusculares e de estresse percebido em docentes universitários em tempos de pandemia da COVID-19.

\section{Metodologia}

Trata-se de um estudo transversal analítico de abordagem quantitativa realizado com o corpo docente dos diversos cursos de graduação de um Centro Universitário no interior de Minas Gerais, atualmente composto por 389 docentes. Utilizouse como critério de inclusão, o fato de o docente estar atuando de forma remota durante a pandemia da COVID-19.

Os profissionais foram contatados por meio de correio eletrônico, explicitando o objetivo da pesquisa e o procedimento de coleta de dados, além da apresentação do Termo de Consentimento Livre e Esclarecido (TCLE). Os docentes tiveram acesso aos instrumentos de coleta de dados apenas após a anuência em participar da pesquisa. Não houve cálculo do tamanho amostral, sendo utilizada uma amostra de conveniência, mediante retorno dos questionários respondidos.

A coleta de dados ocorreu entre os meses de outubro de dezembro de 2020 de forma remota, com auxílio da ferramenta Google Forms®. Para sua efetivação, utilizaram-se três questionários: um questionário sociodemográfico e profissional, a fim de traçar o perfil dos participantes; o Questionário Nórdico de Sintomas Osteomusculares (QNSO), para a avaliação da presença de dores osteomusculares; e a Escala de Estresse Percebido (PSS-10), com o propósito de identificar o estresse percebido pelos docentes.

O QNSO é composto por um mapa corporal dividido em diversas regiões anatômicas, onde o participante relata a ocorrência de sintomas osteomusculares nas diferentes regiões do corpo nos últimos doze meses e nos sete dias anteriores à aplicação do instrumento (Barros \& Alexandre, 2003).

O instrumento PSS-10 avalia a frequência com que o indivíduo sentiu ou pensou de determinada maneira, durante o último mês, mediante cada situação. O instrumento é composto por 10 itens, sendo seis com aspectos positivos e quatro negativos, respondidos em uma escala tipo Likert de frequência, variando de Nunca (0) a Sempre (4) (Reis, Hino \& Añez, 2010).

O escore de estresse percebido foi calculado somando a pontuação de cada item da escala, podendo variar de 0 a 40 . É importante mencionar que os itens 4, 5, 7 e 8 possuem respostas positivas, logo, a pontuação obtida neste item foi revertida antes do cálculo do escore final. Assim, a reposta 0 teve valor 4, 1 teve valor 3, 2 teve valor 2, 3 teve valor 1 e 4 teve valor 0 . Após tal reversão, as pontuações foram somadas normalmente. Para a realização da análise estatística bivariada, utilizou-se a média do escore total como ponto de corte para dicotomizar nos grupos de Percepção do estresse dentro média da amostra e Percepção do estresse acima da média. 
Os dados coletados foram processados no software IBM® SPSS®, versão 21, para realização da análise descritiva e exploratória. Foram calculadas estatísticas descritivas por meio da utilização de medidas resumo de posição (média) e de variabilidade (amplitudes). Utilizou-se também, a análise bivariada por meio das medidas de associação com tabelas de contingência, além da aplicação do teste qui-quadrado de Pearson. Este trabalho considerou um nível de significância de 5\% $(\mathrm{p}<0,05)$.

A pesquisa seguiu as normas estabelecidas pela legislação vigente que rege sobre a realização de pesquisas com seres humanos, sendo aprovada pelo Comitê de Ética em Pesquisa do Centro Universitário do Cerrado Patrocínio (COEP/UNICERP) por meio do parecer $\mathrm{n}^{\circ}$ 20201450FIS005. Os participantes do estudo consentiram participar do estudo mediante assinatura do TCLE, sendo orientados previamente pelo pesquisador quanto aos objetivos da pesquisa e esclarecidos que não seriam submetidos a riscos ou prejuízos, e que poderiam desistir de participar do estudo a qualquer momento. Ainda, para minimizar a exposição dos participantes, os mesmos foram identificados por meio de um código alfanumérico.

\section{Resultados}

A amostra foi constituída por 51 docentes, conforme caracterização apresentada na Tabela 1. Destaca-se que 64,7\% são do sexo feminino; com predominância na faixa etária de 31 a 49 anos (56,9\%); 66,7\% são casados; e 58,8\% possuem filhos. Quanto às características acadêmica e profissional, 41,2\% possuem especialização e mestrado; $82,4 \%$ possuem dois ou mais vínculos empregatícios; $41,2 \%$ realizam uma carga horária de trabalho de 40 horas semanais; 35,3\% recebem de quatro a seis salários mínimos; e 58,8\% dos docentes trabalham na instituição por até cinco anos. 
Research, Society and Development, v. 10, n. 6, e25110615447, 2021

(CC BY 4.0) | ISSN 2525-3409 | DOI: http://dx.doi.org/10.33448/rsd-v10i6.15447

Tabela 1 - Caracterização sociodemográfica e profissional da amostra $(n=51)$.

Patrocínio-MG, 2020.

\begin{tabular}{|c|c|c|}
\hline & $\mathrm{N}$ & $\%$ \\
\hline \multicolumn{3}{|l|}{ Sexo } \\
\hline Feminino & 33 & 64,7 \\
\hline Masculino & 18 & 35,3 \\
\hline \multicolumn{3}{|l|}{ Faixa etária } \\
\hline Até 30 anos & 11 & 21,6 \\
\hline 31 a 49 anos & 29 & 56,9 \\
\hline 50 anos ou mais & 11 & 21,6 \\
\hline \multicolumn{3}{|l|}{ Situação conjugal } \\
\hline Solteiro (a) & 16 & 31,4 \\
\hline Casado (a) & 34 & 66,7 \\
\hline Divorciado (a) ou separado (a) & 1 & 2,0 \\
\hline \multicolumn{3}{|l|}{ Possui filhos? } \\
\hline Sim & 30 & 58,8 \\
\hline Não & 21 & 41,2 \\
\hline \multicolumn{3}{|l|}{ Formação acadêmica } \\
\hline Graduação & 2 & 3,9 \\
\hline Especialização & 21 & 41,2 \\
\hline Mestrado & 21 & 41,2 \\
\hline Doutorado & 7 & 13,7 \\
\hline \multicolumn{3}{|l|}{ Quantidade de vínculos empregatícios } \\
\hline 01 & 9 & 17,6 \\
\hline 02 & 32 & 62,7 \\
\hline 03 & 9 & 17,6 \\
\hline 04 & 1 & 2,0 \\
\hline \multicolumn{3}{|l|}{ Carga horária semanal } \\
\hline Até 20 horas & 2 & 3,9 \\
\hline 20 horas & 5 & 9,8 \\
\hline 30 horas & 7 & 13,7 \\
\hline 40 horas & 21 & 41,2 \\
\hline 60 horas & 13 & 25,5 \\
\hline Mais de 60 horas & 3 & 5,9 \\
\hline \multicolumn{3}{|l|}{ Remuneração recebida* } \\
\hline Até 2 salários & 5 & 9,8 \\
\hline 2 a 4 salários & 15 & 29,4 \\
\hline 4 a 6 salários & 18 & 35,3 \\
\hline 6 a 8 salários & 5 & 9,8 \\
\hline Mais de 8 salários & 8 & 15,7 \\
\hline \multicolumn{3}{|l|}{ Tempo de trabalho na instituição } \\
\hline Até 5 anos & 30 & 58,8 \\
\hline 6 a 10 anos & 10 & 19,6 \\
\hline 11 a 20 anos & 8 & 15,7 \\
\hline 21 anos ou mais & 3 & 5,9 \\
\hline
\end{tabular}

*Salário mínimo vigente no período da coleta de dados. Fonte: Dados da pesquisa (2020).

A distribuição das queixas por sinais e sintomas, por região corporal está descrita na Tabela 2. Percebe-se que a principal queixa osteomuscular dos docentes está localizada na região das costas, enquanto que a queixa menos frequente é o formigamento nos cotovelos, tanto no último ano, quanto na última semana. Percebe-se também que, diante das queixas osteomusculares dos últimos 12 meses, apenas os problemas relacionados aos cotovelos não impediram os docentes de realizarem suas atividades normais (por exemplo, trabalho, atividades domésticas e de lazer) e que os distúrbios osteomusculares localizados na parte inferior das costas foram os que mais fizeram os docentes a buscarem atendimento com profissional de saúde. 
Research, Society and Development, v. 10, n. 6, e25110615447, 2021

(CC BY 4.0) | ISSN 2525-3409 | DOI: http://dx.doi.org/10.33448/rsd-v10i6.15447

Tabela 2 - Distribuição das queixas por sinais e sintomas, por região corporal. Patrocínio-MG, 2020.

\begin{tabular}{|c|c|c|c|c|}
\hline & \multicolumn{2}{|c|}{ Formigamento/ Dormência } & \multirow{2}{*}{$\begin{array}{r}\text { Impedimento } \\
\text { Últimos } 12 \\
\text { meses }(\%) \\
\end{array}$} & \multirow{2}{*}{$\begin{array}{l}\text { Consulta médica } \\
\text { Últimos } 12 \text { meses (\%) }\end{array}$} \\
\hline & $\begin{array}{l}\text { Últimos } 12 \\
\text { meses }(\%)\end{array}$ & $\begin{array}{c}\text { Últimos } 7 \\
\text { dias }(\%)\end{array}$ & & \\
\hline \multicolumn{5}{|c|}{ Pescoço } \\
\hline Sim & 47,1 & 29,4 & 5,9 & 9,8 \\
\hline Não & 52,9 & 70,6 & 94,1 & 90,2 \\
\hline \multicolumn{5}{|c|}{ Ombros } \\
\hline $\operatorname{Sim}$ & 54,9 & 31,4 & 9,8 & 7,8 \\
\hline Não & 45,1 & 68,6 & 90,2 & 92,2 \\
\hline \multicolumn{5}{|c|}{ Parte superior das costas } \\
\hline Sim & 62,7 & 37,3 & 9,8 & 9,8 \\
\hline Não & 37,3 & 62,7 & 90,2 & 90,2 \\
\hline \multicolumn{5}{|c|}{ Cotovelo } \\
\hline Sim & 3,9 & 0 & 0 & 3,9 \\
\hline Não & 96,1 & 100 & 100 & 96,1 \\
\hline \multicolumn{5}{|c|}{ Punhos/ Mãos } \\
\hline Sim & 37,3 & 7,8 & 3,9 & 7,8 \\
\hline Não & 62,7 & 92,2 & 96,1 & 92,2 \\
\hline \multicolumn{5}{|c|}{ Parte inferior das costas } \\
\hline Sim & 11,8 & 33,3 & 13,7 & 21,6 \\
\hline Não & 88,2 & 66,7 & 86,3 & 78,4 \\
\hline \multicolumn{5}{|c|}{ Quadril/ Coxas } \\
\hline Sim & 11,8 & 9,8 & 5,9 & 7,8 \\
\hline Não & 88,2 & 90,2 & 94,1 & 92,2 \\
\hline \multicolumn{5}{|c|}{ Joelhos } \\
\hline Sim & 25,5 & 13,7 & 7,8 & 3,9 \\
\hline Não & 74,5 & 86,3 & 92,2 & 96,1 \\
\hline \multicolumn{5}{|c|}{ Tornozelos/ Pés } \\
\hline Sim & 23,5 & 9,8 & 5,9 & 7,8 \\
\hline Não & 76,5 & 90,2 & 94,1 & 92,2 \\
\hline
\end{tabular}

Fonte: Dados da pesquisa (2020).

Na aplicação da Escala de Estresse Percebido, foram obtidos escores que variaram de 8 a 29, e média de 18,12. Dos 51 docentes, 45,1\% apresentaram escores acima da média encontrada. Os escores de estresse percebido acima da média apresentaram associação estatisticamente significativa com a presença de distúrbios osteomusculares no pescoço ( $\mathrm{p}=0,02)$ e ombros $(0,05)$, nos últimos 12 meses, conforme descrito na Tabela 3. 
Tabela 3 - Associação do escore de estresse percebido com a presença de distúrbios osteomusculares, nos últimos 12 meses e por região corporal. Patrocínio-MG, 2020.

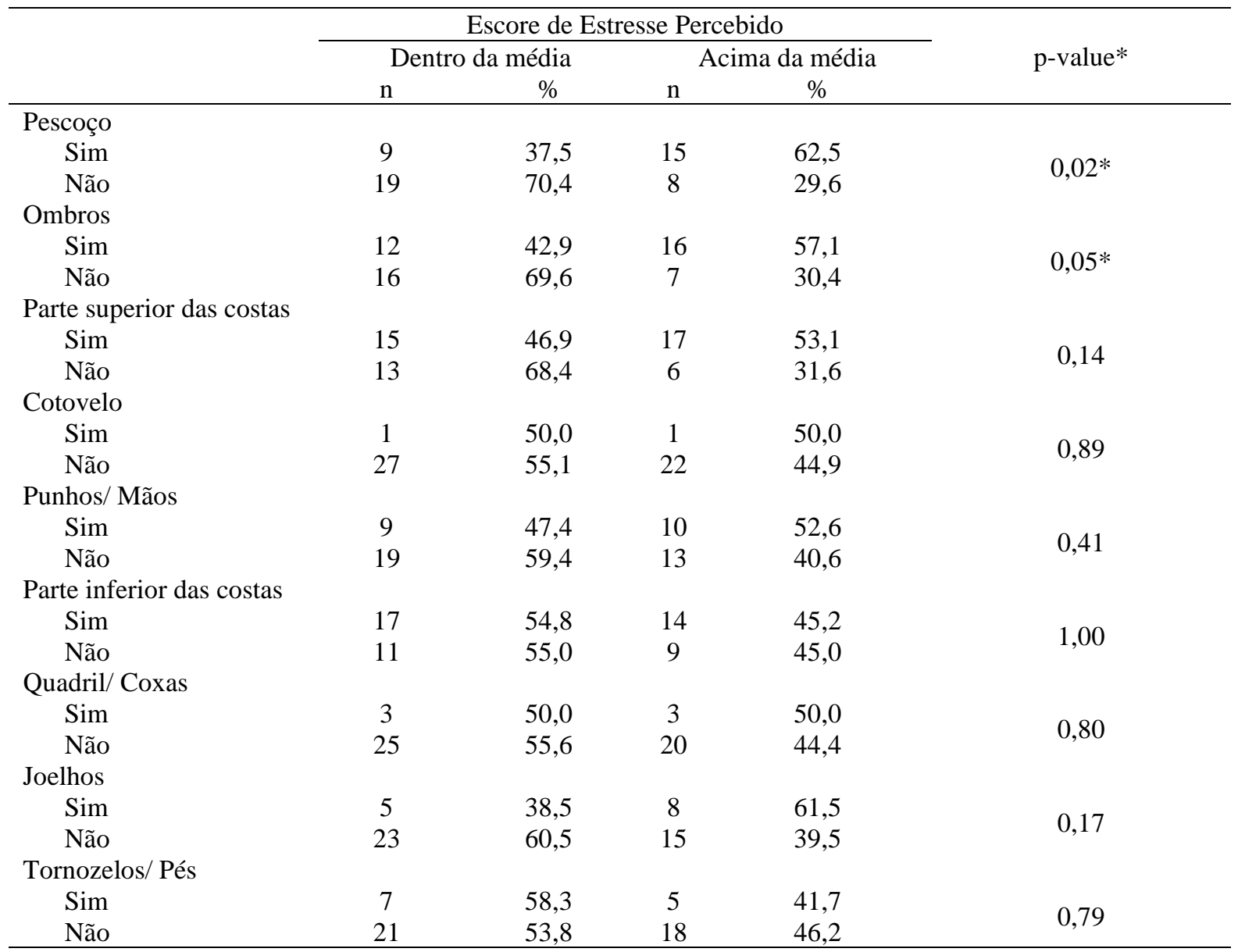

* p-value: Nível de significância ( $\mathrm{p}<0,05)$ Fonte: Dados da pesquisa (2020).

Não se observou associação estatisticamente significativa entre o escore de estresse percebido e o impedimento da realização das atividades normais por problemas osteomusculares, nem entre o escore de estresse percebido e a busca de consulta com profissional de saúde por problemas osteomusculares, como é mostrado nas tabelas 4 e 5, respectivamente. 
Tabela 4 - Associação do escore de estresse percebido com o impedimento da realização das atividades normais por problemas osteomusculares, nos últimos 12 meses, por região corporal. Patrocínio-MG, 2020.

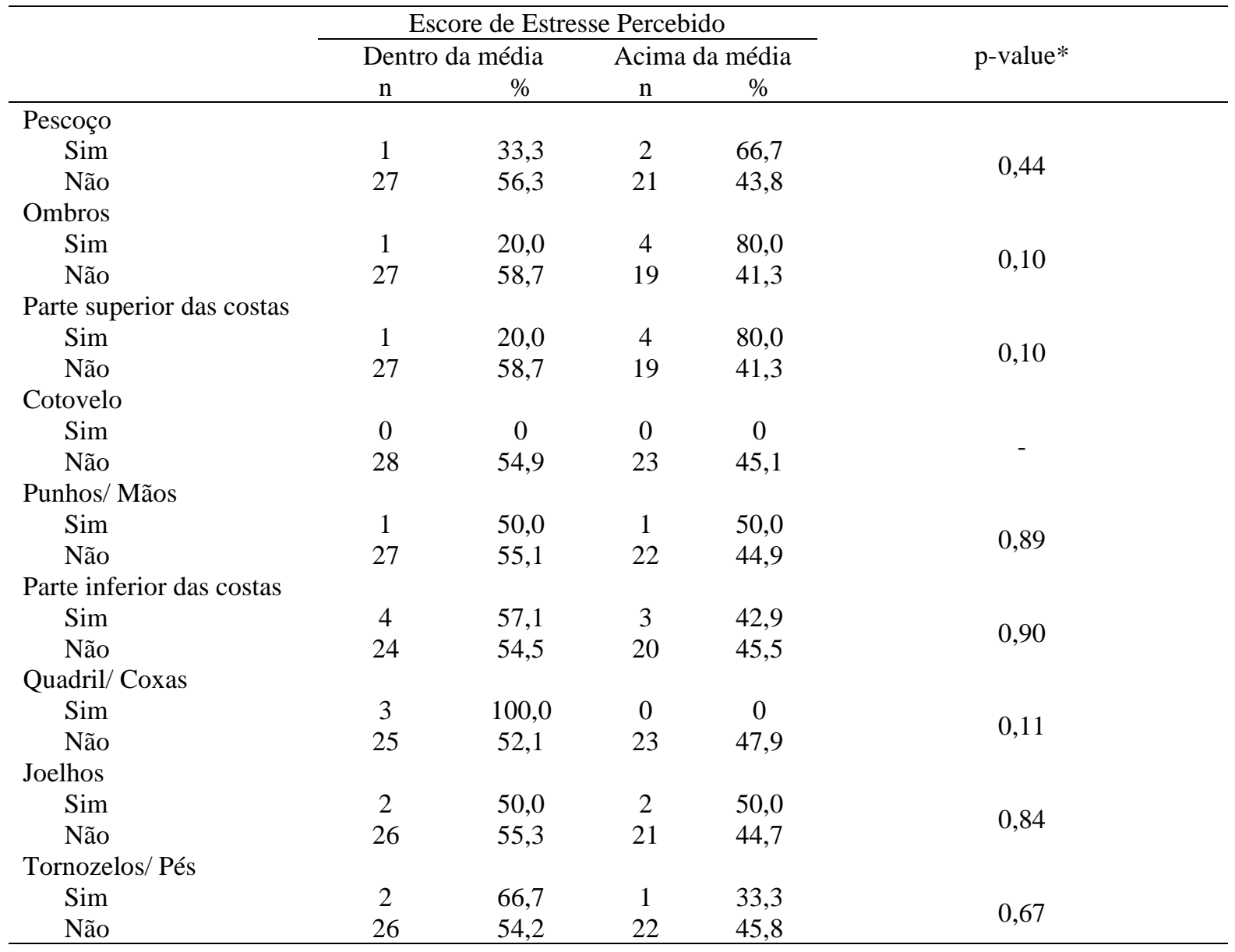

* p-value: Nível de significância (p<0,05). Fonte: Filippo (2017). 
Tabela 5 - Associação do escore de estresse percebido com a procura de consulta com profissional de saúde por problemas osteomusculares, nos últimos 12 meses, por região corporal. Patrocínio-MG, 2020.

\begin{tabular}{|c|c|c|c|c|c|}
\hline & \multicolumn{4}{|c|}{ Escore de Estresse Percebido } & \multirow{3}{*}{ p-value* } \\
\hline & \multicolumn{2}{|c|}{ Dentro da média } & \multicolumn{2}{|c|}{ Acima da média } & \\
\hline & $\mathrm{n}$ & $\%$ & $\mathrm{n}$ & $\%$ & \\
\hline \multicolumn{6}{|l|}{ Pescoço } \\
\hline Sim & 1 & 20,0 & 4 & 80,0 & \multirow{2}{*}{0,10} \\
\hline Não & 27 & 58,7 & 19 & 41,3 & \\
\hline \multicolumn{6}{|l|}{ Ombros } \\
\hline Sim & 1 & 25,0 & 3 & 75,0 & \multirow{2}{*}{0,21} \\
\hline Não & 27 & 57,4 & 20 & 42,6 & \\
\hline \multicolumn{6}{|c|}{ Parte superior das costas } \\
\hline $\operatorname{Sim}$ & 2 & 40,0 & 3 & 60,0 & \multirow{2}{*}{0,48} \\
\hline Não & 26 & 56,5 & 20 & 43,5 & \\
\hline \multicolumn{6}{|l|}{ Cotovelo } \\
\hline Sim & 0 & 0 & 2 & 100,0 & \multirow{2}{*}{0,11} \\
\hline Não & 28 & 57,1 & 21 & 42,9 & \\
\hline \multicolumn{6}{|c|}{ Punhos/ Mãos } \\
\hline Sim & 1 & 25,0 & 3 & 75,0 & \multirow{2}{*}{0,21} \\
\hline Não & 27 & 57,4 & 20 & 42,6 & \\
\hline \multicolumn{6}{|c|}{ Parte inferior das costas } \\
\hline Sim & 6 & 54,5 & 5 & 45,5 & \multirow{2}{*}{0,98} \\
\hline Não & 22 & 55,0 & 18 & 45,0 & \\
\hline \multicolumn{6}{|c|}{ Quadril/ Coxas } \\
\hline $\operatorname{Sim}$ & 2 & 50,0 & 2 & 50,0 & \multirow{2}{*}{0,84} \\
\hline Não & 26 & 55,3 & 21 & 44,7 & \\
\hline \multicolumn{6}{|l|}{ Joelhos } \\
\hline Sim & 0 & 0 & 2 & 100,0 & \multirow{2}{*}{0,11} \\
\hline Não & 28 & 57,1 & 21 & 42,9 & \\
\hline \multicolumn{6}{|c|}{ Tornozelos/ Pés } \\
\hline Sim & 3 & 75,0 & 1 & 25,0 & \multirow{2}{*}{0,40} \\
\hline Não & 25 & 53,2 & 22 & 46,8 & \\
\hline
\end{tabular}

* p-value: Nível de significância (p<0,05). Fonte: Filippo (2017).

\section{Discussão}

O perfil sociodemográfico observado neste estudo evidencia a presença majoritária de docentes do sexo feminino e que a maioria delas busca cada vez mais a capacitação profissional. Vários estudos corroboram com este achado, indicando que o docente está comprometido com a qualidade do ensino ofertada (Almeida \& Dumith, 2018; Araújo, Gomes, Pires, Moraes Filho \& Costa, 2015; Silva, Ferreira \& Mattos, 2018).

Espera-se que quanto maior o nível de capacitação melhor a atuação no cenário docente, principalmente quanto ao aspecto de conhecimento e da utilização de diversas metodologias que possibilitam a construção e troca de saberes. Ademais, a crescente qualificação colabora para o aumento da autossatisfação e aprendizado dos docentes (Sousa, et al., 2018). No entanto, Dalagasparenia e Monteiro (2016) descrevem que quanto mais elevado o nível de qualificação do docente, mais a categoria estará predisposta ao estresse ocupacional, devido às obrigações e responsabilidades inerentes ao cargo ocupado.

Em estudo desenvolvido numa universidade pública do município do Rio de Janeiro, D’Oliveira et al. (2018) relataram que a sobrecarga de atividades do docente desencadeia diversas consequências negativas na saúde destes profissionais, expressadas por meio do estresse, do cansaço e da sobrecarga emocional. Apreenderam-se também impactos negativos na dimensão social do docente como a perda do tempo de lazer e de convívio com a família, e a invasão do tempo de descanso do trabalhador a fim de atender as expectativas da organização laboral. 
Outro aspecto relevante ao analisar o nível de estresse está relacionado ao estado civil dos docentes. Pessoas casadas ou conviventes em união estável encontram-se, na maioria das vezes, em um ambiente com um maior apoio emocional, afetivo e social, principalmente em famílias estruturadas e com filhos. Pertencem a um grupo considerado mais estável no quesito emocional, prudentes e com mais discernimento ao enfrentar situações problemáticas, contribuindo para a redução da incidência de estresse e/ou doenças ocupacionais (Soares, Mafra \& Faria, 2018; Amaro \& Dumith, 2018).

O número de vínculos empregatícios nesse estudo apresentou-se elevado, indicando que o profissional docente busca a realização de outra atividade profissional na carreira, para complementação de renda ou por satisfação pessoal. É indubitável que o acúmulo de atividades laborais acarreta uma sobrecarga trabalhista, principalmente para os docentes por ser uma função que exige preparo de materiais, organização de aulas, elaboração e correção de provas e trabalhos, dentre outras funções da carreira docente. A sobrecarga trabalhista, por si só, ocasiona um aumento no nível de estresse dos docentes, tornando o trabalho cansativo e, em alguns casos, prejudicial à saúde (Kirchhof, et al., 2015; Soares, 2016).

Durante a pandemia foi necessário a readaptação das atividades laborais docentes para a modalidade de ensino remoto. Nessa modalidade, foi necessária a reprogramação das atividades a serem desenvolvidas a fim de evitar o desconforto físico e mental, além do aumento da carga trabalhada. Ressalta-se o surgimento da tensão e do estresse ocasionado pelo acúmulo de atividades, tanto domésticas quanto profissionais, principalmente por parte das mulheres, causados pelas incertezas vivenciadas no momento atual, resultando em fadiga corporal, como o aparecimento de dores nas costas (Neves, 2020).

Em estudo semelhante ao presente, Kraemer, Moreira e Guimarães (2020) observaram que todos os docentes estudados apresentaram dor nos últimos 12 meses. Ainda segundo esses autores, as regiões corporais com maior prevalência foram coluna lombar (60\%), pescoço (56\%) e ombros (48\%). Almeida e Dumith (2018) também encontraram resultados semelhantes, observando que a região das costas foi a mais prevalente de dor. Tais dados corroboram com os achados do estudo em tela e reforça a necessidade da implantação de ações específicas e preventivas ao surgimento de distúrbio musculoesquelético em docentes.

Em investigação realizada na Turquia fez-se a comparação entre um grupo de 375 indivíduos, dentre eles professores, que permaneceram em casa e outro de 311 indivíduos que trabalharam em seu ambiente de ocupação habitual, durante o bloqueio de três meses no país, devido ao período pandêmico. A pesquisa revelou a presença de dor lombar elevada no grupo que ficou em casa (Celenay, Karaaslan, Mete \& Kaya, 2020). O comportamento sedentário pode estar relacionado à sobrecarga da coluna lombar resultando em queixas álgicas (Mahdavi \& Kelishadi 2020). Também corroborando com este estudo.

É importante mencionar que a dor, mesmo de forma não tão intensa ou apenas constante por certo tempo, tende a afetar diretamente situações rotineiras de uma pessoa, ocasionando incapacidades e afetando o bem estar. A dor lombar pode ocorrer por adoção de posturas incorretas e não confortáveis por um longo período. Ainda que sua resolução seja comumente voluntária, um excesso de prazo irá ocasionar uma lombalgia crônica diante da degeneração discal intervertebral sofrida (Bento, Paiva \& Siqueira, 2009).

Cabe salientar que, na realização do ensino remoto, os profissionais têm desempenhado suas atividades laborais no domicílio e, muitas vezes, não há uma infraestrutura adequada prejudicando a ergonomia e a disposição física dos docentes. A reestruturação do trabalho trouxe à tona a precariedade das condições laborais, somadas ao aumento da sobrecarga relacionada com a extensividade da jornada, falta de descanso e lazer dos docentes, queixas de sofrimento e doenças mentais e físicas (Rodrigues, Souza, Teixeira \& Larentis, 2020).

A postura sentada pode ocasionar alterações na coluna lombar, especificamente na condição musculoesquelética, ocasionando desequilíbrio em seus componentes. Como resultado, ligamentos, pequenas articulações, nervos e todas as estruturas em plano posterior da região são distendidas, ocorrendo um crescimento de aproximadamente 35\% na pressão intradiscal no momento em que o indivíduo vai da postura de pé para sentada, ainda que tal postura se dê da maneira mais correta possível. 
Não obstante, quando na postura sentada por um longo período de uma maneira incorreta, tal percentual irá corresponder a mais de 70\% a pressão sob o núcleo do disco intervertebral. Como consequências o indivíduo poderá ter maiores desconfortos como, por exemplo, sensação de formigamento e peso no corpo, predisposição a degenerações e/ou ocorrência de dores (Zapater, et al., 2004).

No último ano, em decorrência da pandemia COVID-19, os docentes tiveram que se reinventar para se adaptarem à nova forma de lecionar, através do trabalho remoto, tendo que utilizar tecnologias, recursos e plataformas de ensino em sua maioria desconhecidos, gerando cada vez mais ansiedade, nervosismo e estresse nos docentes. O estudo de Souza, Mendonça, Rodrigues, Felix, Teixeira, Santos e Moura (2017) alerta para a necessidade de promoção à saúde dos docentes, propondo a inserção de espaços de apoio para o diálogo de ansiedades e outras questões de saúde.

A angústia vivenciada torna-se ainda maior quando levamos em conta o fato de ser um vírus totalmente novo, desconhecido e imprevisível e ainda por não ser possível determinar o tempo de duração do cenário vivenciado. Um estudo na Turquia mediu o nível de coronafobia de indivíduos que trabalhavam em casa, como professores e estudantes e dos que, mesmo em pandemia, tiveram que ir para o ambiente de trabalho como médicos e outros profissionais de setores privados. Os participantes que estiveram em casa revelaram índices mais altos para coronafobia (Celenay, Karaaslan, Mete \& Kaya, 2020). Esta condição está relacionada ao aumento da ansiedade, o medo da perda de entes queridos e elevada exposição às redes sociais (Mertens, Gerritsen, Duijndam, Salemink \& Engelhard, 2020).

O corpo é reflexo dos sentimentos onde a tensão, ansiedade e estresse vividos diariamente acabam por colaborar com o aumento das dores osteomusculares. Como visto apenas as queixas relacionadas aos cotovelos não impediram os docentes de realizarem outras atividades rotineiras.

Alguns estudos demonstram uma relação entre aqueles que sentem dores musculoesqueléticas e as percepções de estresse, onde o primeiro pode inferir subjetivamente no segundo, talvez pelo fato da dor ser mais sensível àqueles com alterações psicológicas resultantes da atividade laboral, seja ela qual for (Bonzini, Bertu, Veronesi, Conti, Coggon, \& Ferrario, 2015).

Assim, a exposição prolongada a condições de trabalho desfavoráveis como da maioria dos docentes em trabalho remoto devido à pandemia COVID-19, representa fatores de risco à saúde, levando-os a procurarem ajuda com profissionais da saúde por problemas osteomusculares, principalmente no que tange à região das costas, como identificada nesse estudo.

Reconhece-se que o estresse desencadeia sintomas orgânicos fisiologicamente tóxicos, estando associado a desconfortos que alteram o equilíbrio corporal. Estudiosos veem o estresse como um sinal de alarme do corpo que se aproxima da exaustão (Almeida \& Dumith, 2018; Farias, Teixeira, Moreira, Oliveira \& Pereira, 2011).

Dessa forma, a percepção de estresse acima da média por $45,1 \%$ dos professores demonstra que tais profissionais podem estar expostos a situações desgastantes, capazes de gerar desconforto físico e mental (Fernandes \& Vandenbergue, 2018). Neste cenário, torna-se desafiador o desempenho da atividade docente. Algumas situações como a inflexibilidade de horários, indisciplina em sala de aula, precariedade das condições existentes, burocracia administrativa, déficit de recursos, situação salarial, entre outras adversidades, acarreta em sobrecarga de trabalho (Luz, et al., 2019).

Os escores de estresse percebido encontrados na literatura são bem variados, haja vista que a média de percepção do estresse depende de características intrínsecas à amostra estudada. Em estudo realizado com profissionais de uma escola pública na região norte do Brasil, obteve-se média de percepção do estresse de 17,65, semelhante ao encontrado no estudo em tela (Silva, Barbosa, Cantanhêde, Quaresma \& Maciel, 2017). Em contrapartida, Almeida e Dumith (2018) encontraram média do escore de estresse percebido de 42,8, num inquérito realizado na região sul do Brasil.

Um estudo realizado com 304 docentes de um hospital na Índia, mostrou que 31\% relatou níveis altos de estresse no trabalho. O estresse foi inversamente associado à satisfação no trabalho e associado significativamente ao burnout (Chichra, Abhijnhan \& Tharyan, 2019). 
As novas formas de desenvolvimento do trabalho docente em tempos de pandemia revelaram outros sintomas osteomusculares entre os docentes, saindo da alta prevalência de sintomas lombares que desencadeavam a necessidade de atendimento de profissional de saúde ou limitavam a realização das atividades docentes, para os sintomas na região do pescoço e ombros. Ademais, a associação de estresse percebido e a presença de distúrbio osteomuscular no pescoço e ombros são corroboradas por estudo realizado com servidores de uma instituição de ensino superior no sul do Brasil (Almeida \& Dumith, 2018). Tais autores relatam, ainda, que quanto maior o estresse percebido, maior a prevalência de sintomas osteomusculares.

\section{Conclusão}

Observou-se que a maior parte dos docentes que atuam de forma remota durante a pandemia da COVID-19, são mulheres jovens, casadas, com filhos, com renda acima da média nacional, com mais de um emprego, sendo especialistas ou mestres na sua área de atuação.

O período de atividade remota aponta uma percepção de estresse acima da média em 45,1\% os participantes, estando diretamente relacionada à existência de distúrbios osteomusculares no pescoço e ombros nos últimos 12 meses. Neste período, apenas os problemas relacionados aos cotovelos não impediram os docentes de realizarem suas atividades normais e os distúrbios osteomusculares localizados na parte inferior das costas foram os que mais fizeram os docentes a buscarem atendimento com profissional de saúde.

Algumas limitações devem ser consideradas na interpretação dos resultados do estudo em tela. A generalização dos achados é limitada, uma vez que a coleta de dados foi restrita a uma única instituição de ensino e houve uma baixa adesão da população em participar do estudo. Outra questão diz respeito ao delineamento transversal do estudo, em que há a limitação da causalidade. Além disso, percebeu-se uma escassez de evidências científicas na literatura quanto à associação de estresse percebido e a presença de distúrbio osteomuscular. Ressalta-se, portanto, a importância da realização de novos estudos na temática a fim de investigar fatores de causalidade e explicitar a relação dos achados.

\section{Referências}

Almeida, L. M. S., \& Dumith, S. C. (2018). Association between musculoskeletal symptoms and perceived stress in public servants of a Federal University in the South of Brazil. Br J Pain, 1 (1), 9-14.

Amaro, J. M. R. S., \& Dumith, S.C. (2018). Sonolência diurna excessiva e qualidade de vida relacionada à saúde dos professores universitários. J Bras Psiquiatr., 67 (2), 94-100.

Araújo, B. L. S., Gomes, D. V., Pires, V. S., Moraes Filho, I. M., \& Costa, A. L. S. (2015). Estresse ocupacional em docentes de uma instituição de ensino superior da região metropolitana de Goiânia. REVISA, 4 (2), 96-104.

Barros, E. N. C., \& Alexandre, N. M. C. (2003). Cross- cultural adaptation of the Nordic musculoskeletal questionnaire. International nursing review, 50 (2), 101-108.

Bento, A. A. C., Paiva, A. C. S., \& Siqueira, F. B. (2009). Correlação entre incapacidade, dor - Roland Morris, e capacidade funcional - SF-36 em indivíduos com dor lombar crônica não específica. E-scientia, 2 (1).

Bonzini, M., Bertu, L., Veronesi, G., Conti, M., Coggon, D., Ferrario, M. M. (2015). Is musculoskeletal pain a consequence or a cause of occupational stress? A longitudinal study. Arch Occup Environ Health, 88(5):607-12.

Brasil. (2020). Ministério da Saúde. Painel Coronavírus (COVID - 19). https://covid.saude.gov.br.

Celenay, S. T., Karaaslan, Y., Mete, O., \& Kaya, D. O. (2020). Coronaphobia, musculoskeletal pain, and sleep quality in stay-at home and continuedworking persons during the 3-month Covid-19 pandemic lockdown in Turkey, Chronobiology International, 37(12), 1778-1785.

Chichra, A., Abhijnhan, A., \& Tharyan, P. (2019). Job stress and satisfaction in faculty of a teaching hospital in south India: A cross-sectional survey. J Postgrad Med., 65 (4), 201-206.

Dalagasperina, P., \& Monteiro, J. K. (2016). Estresse e docência: um estudo do ensino superior privado. Revista Subjetividades, 16 (1), 37-51.

D’Oliveira, C. A. F. B., Almeida, C. M., Souza, N. V. D. O., Pires, A., Madriaga, L. C. V., \& Varella, T. C. M. M. L. (2018). Teaching work of nursing and the impact on the health-disease process. Rev Fund Care Online, 10 (1), 196-202. 
Farias, S. M. C., Teixeira, O. L. C., Moreira, W., Oliveira, M. A. F., Pereira, M. O. (2011). Characterization of the physical symptoms of stress in the emergency health care team. Rev Esc Enferm USP, 45(3):722-9.

Fernandes, G. C. P. S., \& Vandenbergue, L. (2018). O estresse, o professor e o trabalho docente. Revista Labor, 1 (19), 75-86.

Freitas, A. R. R., Napimoga, M., \& Donalisio, M. R. (2020). Análise da gravidade da pandemia de COVID-19. Epidemiol. Serv. Saúde, 29 (2), 1-5.

Kirchhof, R. S., Freitas, E. O., Silva, R. M., Guido, L. A., Costa, A. L. S., \& Lopes, L. F. D. (2015). Relations between stress and coping in federal universities nursing teachers of a Brazilian state-analytical study. J Nurs Educ Pract., 5 (12), 9-16.

Kraemer, K., Moreira, M. F., \& Guimarães, B. (2020). Dor musculoesquelética e riscos ergonômicos em docentes de uma instituição federal. Rev Bras Med Trab, 18 (3), 343-351.

Luz, J.G., Pessa, S. L. R., Luz, R. P., \& Schenatto, F. J. A. (2019). Implicações do ambiente, condições e organização do trabalho na saúde do professor: uma revisão sistemática. Ciência e Saúde coletiva, 24 (12), 4621-4632.

Mahdavi, S.B., \& Kelishadi, R. (2020). Impact of sedentary behavior on bodily pain while staying at home in COVID-19 pandemic and potential preventive strategies. Asian J Sports Med., 11(2):e103511.

Mertens, G., Gerritsen, L., Duijndam, S., Salemink, E., \& Engelhard, I. M. (2020). Fear of the coronavirus (COVID-19): predictors in an online study conducted in March. J Anxiety Disord. 74:102258.

Monteiro, B. M. M., \& Souza, J. C. (2020). Mental health and university teaching working conditions in the COVID 19 pandemic. Research, Society and Development, 9 (9).

Neves, C. O. (2020). Vírus da desigualdade - Os Impactos do COVID-19 na vida da mulher. Revista Jus Navigandi.

Portaria $n^{\circ}$ 544, de 16 de junho de 2020. (2020). Dispõe sobre a substituição das aulas presenciais por aulas em meios digitais, enquanto durar a situação de pandemia do novo coronavírus - Covid-19, e revoga as Portarias MEC nº 343, de 17 de março de 2020, nº 345, de 19 de março de 2020, e n 473 , de 12 de maio de 2020. https://www.in.gov.br/en/web/dou/-/portaria-n-544-de-16-de-junho-de-2020-261924872.

Reis, R. S., Hino, A. A. F., Añez, C. R. R. (2010). Perceived Stress Scale: Reliability and Validity Study in Brazil. Journal of Health Psychology, ;15 (1), $107-$ 114.

Rodrigues, A. M. dos S., Souza, K. R. de, Teixeira, L. R., \& Larentis, A. L. (2020). A temporalidade social do trabalho docente em universidade pública e a saúde. Ciência \& Saúde Coletiva, 25 (5), 1.829-1.838.

Silva, B. K. R., Barbosa, T. C., Cantanhêde, L. S., Quaresma, F. P. R., \& Maciel, E. S. (2017). Fatores associados à percepção da qualidade de vida e estresse em funcionários de uma escola pública no Brasil. Revista CPAQV - Centro de Pesquisas Avançadas em Qualidade de Vida, 9 (1).

Silva, N. S. R., Ferreira, D. S., \& Mattos, J. G. S. (2018). Análise do estresse ocupacional em docentes da área da saúde de uma instituição particular do interior de Minas Gerais. Revista Educação, Saúde e Meio Ambiente, 1 (3), 10-24.

Soares, M. B. (2016). Fatores determinantes para a ocorrência do estresse em docentes universitários. Análise do estresse ocupacional em docentes da Universidade Federal de Viçosa e suas interferências na qualidade de vida e suporte familiar. Dissertação (mestrado) - Universidade Federal de Viçosa, Viçosa, MG, Brasil.

Soares, M. B., Mafra, S. C. T., \& Faria, E. R. (2018). A relação entre a carreira do magistério superior, suporte familiar e estresse ocupacional dos docentes da Universidade Federal de Viçosa-MG. Textos \& Contextos, 17 (2), 321-334.

Sousa, A. R., Santos, R. B., Silva, R. M, Santos, C. C. T., Lopes, V. C., \& Mussi, F. C. (2018). Estresse ocupacional e qualidade do sono em docentes da área da saúde. Rev Rene., 19 (e33088), 1-8.

Souza, K.R., Mendonça, A.L.O., Rodrigues, A.M.S., Felix, E.G., Teixeira, L.R., Santos, M.B.M., \& Moura, M. (2017). A nova organização do trabalho na universidade pública: consequências coletivas da precarização na saúde dos docentes. Ciência \& Saúde Coletiva, 22(11), 3667-3676.

Tostes, M. V., Albuquerque, G. S. C., Silva, M. J. S., \& Petterle, R. R. (2018). Sofrimento mental de professores do ensino público. Saúde em Debate, 42 (116), 87-99.

WHO (2021). World Health Organization. COVID-19, situation reports. https://covid19.who.int/table.

Zapater, A. R., Silveira, D. M., Vitta, A., Padovani, C. R., \& Silva, J. C. P. (2004). Postura sentada: a eficácia de um programa de educação para escolares. Ciência \& Saúde Coletiva, 9 (1), 191-199. 